\title{
Interstellar extinction in the California Nebula region
}

\author{
V. Straižys ${ }^{1}$, K. Černis ${ }^{1}$, and S. Bartašiūtè $\dot{e}^{1,2}$ \\ 1 Institute of Theoretical Physics and Astronomy, Goštauto 12, Vilnius 2600, Lithuania \\ 2 Vilnius University Observatory, Čiurlionio 29, Vilnius 2009, Lithuania
}

Received 15 September 2000 / Accepted 17 April 2001

\begin{abstract}
Vilnius seven-color photometry has been obtained for 238 stars down to $\sim 13$ mag in the area of the California Nebula in Perseus. For nearly all of the stars, photometric spectral classes, luminosity classes, absolute magnitudes, interstellar reddenings, extinctions and distances are determined. The "extinction versus distance" diagrams give evidence for the presence of one dust layer at $\sim 160 \mathrm{pc}$ distance in the direction of the California Nebula and its nearest surroundings and of two dust layers at distances of $160 \mathrm{pc}$ and 300 pc northwest of the nebula, in the direction of the dark clouds L 1449 and L 1456. The front layer contributes extinction between 0.3 and $1.3 \mathrm{mag}$, and the second layer gives about $1 \mathrm{mag}$ of additional extinction. It appears likely that the front dust layer is the extension of the Taurus dark clouds. The second dust layer probably belongs to the complex of dark clouds found in other areas of the southern part of Perseus (in the directions of the open cluster IC 348, the reflection nebula NGC 1333, etc.). Both cloud complexes run more or less parallel to the Galactic plane.
\end{abstract}

Key words. methods: observational - techniques: photometric - stars: fundamental parameters - interstellar medium: dust, extinction - nebulae: individual: California Nebula (or NGC 1499)

\section{Introduction}

The complex of dark clouds in the Taurus, Auriga, Perseus and Aries constellations is one of the largest concentrations of gas and dust in the Galaxy. It extends $\sim 25^{\circ}$ parallel to the Galactic equator, which is equal to $61 \mathrm{pc}$ at the $130 \mathrm{pc}$ distance or $84 \mathrm{pc}$ at the $180 \mathrm{pc}$ distance, these distances corresponding to its front edge in different directions. The depth of the complex is probably of the same order, i.e. $60-80$ pc. In many parts of the clouds, stars illuminate the dust, forming reflection nebulae (e.g. the Pleiades, the NGC 1333 nebula, etc.). In some areas, the ultraviolet radiation of hot luminous stars ionizes the gaseous component of the clouds, forming emission nebulae (like the California Nebula). Radio observations reveal a number of molecular clouds hidden in the densest dust concentrations. According to Ungerechts \& Thaddeus (1987) the total mass of all clouds in the complex is about $2 \times 10^{5} M_{\odot}$. That paper also gives a map of the complex with the molecular clouds, dust clouds, nebulae and clusters identified.

In the complex, star forming processes are taking place: in its eastern section (Taurus and Auriga) mostly

Send offprint requests to: V. Straižys,

e-mail: straizys@itpa.lt low-mass $\mathrm{T}$ Tauri type stars are observed, while in the western section of the complex (Perseus) both low- and high-mass stars are being formed (the Per OB2 association, the open cluster IC 348 , etc.).

In the seventies, we started a program of investigation of this complex of interstellar dust clouds by means of photoelectric photometry of stars in the Vilnius sevencolor system. The following areas have been investigated: the Taurus area containing the Lynds dark clouds L 1538, L 1528, L 1521 and L 1495 (Straižys \& Meištas 1980; Straižys 1982; Straižys et al. 1982a, 1982b, 1985), the Taurus area containing the Lynds dark clouds L 1551, L 1546 and L 1543 (Meištas \& Straižys 1981), the area around the variable star RV Tau (Straižys \& Meištas 1981), the Merope dark cloud in the Pleiades (Černis 1987), the area around the reflection nebula NGC 1333 (Črnis 1990), the areas around the open cluster IC 348 and in the Per OB2 association (Černis 1993), the area around the open clusters NGC 1750 and NGC 1758 (Straižys et al. 1992). In these publications the distance vs. extinction relationships, cloud distances, densities and, in some cases, the extinction laws of the dust clouds were investigated. We concluded that almost everywhere in the Taurus and Perseus sections of the complex, the extinction begins to increase sharply at $130-180$ pc. In the Perseus 
section, evidence was found for the presence of a second dark cloud at 230-270 pc. A model of the spatial distribution of Taurus and Perseus objects was discussed by Černis (1993).

The present paper extends the research of the Perseus clouds in the direction of the California Nebula (NGC 1499) which is closer to the Galactic equator than the areas investigated earlier. The area is bounded by the following 2000.0 coordinates: RA from $3^{\mathrm{h}} 48^{\mathrm{m}}$ to $4^{\mathrm{h}} 10^{\mathrm{m}}$ and DEC from $+35^{\circ}$ to $+39^{\circ}$. The northern part of this area is covered by the dark cloud Khavtassi 257 (Khavtassi 1960). In the Lynds (1962) catalog, several smaller dark clouds, L 1449, L 1456 and L 1459, are separated in the area.

The area includes several condensations of the molecular cloud situated north of California. This molecular cloud was investigated by Elmegreen \& Elmegreen (1978), Wouterloot \& Habing (1985), Ungerer et al. (1985), Ungerechts \& Thaddeus (1987) and Herbertz et al. (1991) in the $\mathrm{OH}$ and $\mathrm{CO}$ radio lines.

The California Nebula itself is an emission region which covers an area of $1 \times 4$ square degrees near the northern boundary of the Per OB2 association. The source of its ionization is the runaway star $\xi$ Per (O 7.5 III, $V=4.04 \mathrm{mag}$ ).

\section{Observations, reductions and quantification of stars}

Photoelectric photometry of 238 stars in the area was performed in the standard passbands of the Vilnius system (Table 1) in 1994-1996 with the $1 \mathrm{~m}$ telescope of the Institute of Theoretical Physics and Astronomy (Vilnius, Lithuania) situated at the Maidanak Observatory in Uzbekistan. In the area, almost all stars down to $V=$ 10 mag and many stars down to $V=11$ mag and even fainter magnitudes were measured. In the dark clouds north of California we observed a number of fainter stars down to $13 \mathrm{mag}$ (but not all). The typical accuracy of magnitudes and color indices is better than $\pm 0.01 \mathrm{mag}$ ( $\mathrm{rms}$ error), but for the faintest stars the accuracy can be somewhat lower, especially in the ultraviolet filters $U$ and $P$ for red or reddened stars.

The extinction coefficients of the atmosphere for every moment of observation were determined by the Nikonov (1976) method adjusted to the Vilnius system by Zdanavičius $(1975,1996)$. Transformation equations from the instrumental to the standard system were determined from observations of about 20 stars in the Cygnus Standard Region (Zdanavičius \& Černienè 1985). Details on the instrumentation and reduction procedures are given by Bartašiūte (1999) and Straižys et al. (2001, hereafter Paper I).

The catalog of $V$ magnitudes and color indices in the standard Vilnius system for the observed stars is published in Paper I.
Table 1. Mean wavelengths and half-widths of passbands of the Vilnius photometric system.

\begin{tabular}{lccccccc}
\hline Passband & $U$ & $P$ & $X$ & $Y$ & $Z$ & $V$ & $S$ \\
\hline$\lambda(\mathrm{nm})$ & 345 & 374 & 405 & 466 & 516 & 544 & 656 \\
$\Delta \lambda(\mathrm{nm})$ & 40 & 26 & 22 & 26 & 21 & 26 & 20 \\
\hline
\end{tabular}

For the determination of spectral classes and absolute magnitudes (we call this process the photometric quantification) two independent methods were used.

(1) Interstellar reddening-free diagrams $Q_{U P Y}, Q_{P Y V}$; $Q_{U P Y}, Q_{X Y V} ; Q_{U X Y}, Q_{U P Y V} ; Q_{U P Y}, Q_{X Z S}$ and $Q_{X Z S}$, $Q_{X Y Z}$ calibrated in terms of MK spectral classes and absolute magnitudes $M_{V}$ by Straižys et al. (1982c). The reddening-free $Q$-parameters are defined by the equation:

$Q_{123}=\left(m_{1}-m_{2}\right)-\left(E_{12} / E_{23}\right)\left(m_{2}-m_{3}\right)$,

or

$Q_{1234}=\left(m_{1}-m_{2}\right)-\left(E_{12} / E_{34}\right)\left(m_{3}-m_{4}\right)$,

where

$E_{k, \ell}=\left(m_{k}-m_{\ell}\right)_{\text {reddened }}-\left(m_{k}-m_{\ell}\right)_{\text {intrinsic }}$.

In calculating the $Q \mathrm{~s}$, we used the color excess ratios corresponding to the normal interstellar extinction law. The arguments for the normality of the extinction law in the investigated area are described in Paper I. This method of quantification gives the accuracy of spectral class of the order of \pm 1 decimal spectral subclass. The accuracy of $M_{V}$ depends on spectral type and varies from \pm 0.3 to \pm 0.5 mag. The absolute magnitudes are adjusted to the modern distance scale based on the Hyades distance modulus $V-M_{V}=3.3 \mathrm{mag}$ (Perryman et al. 1998).

(2) The $\sigma Q$-method of matching 14 different reddening-free $Q$-parameters of a program star to those of about 7000 standard stars of various spectral and luminosity classes, metallicities and peculiarity types. This method does not give quantitative values of stellar parameters but selects some standard stars which have a set of $Q$-parameters most similar to those of the program star. If the $\sigma Q$ value is sufficiently low (i.e. the $Q$ differences between the program and the standard star are small), the parameters of the closest standard star may be prescribed to the program star. For photometry of Population I stars with the $1 \%$ accuracy, $\sigma Q$ is usually of the order of $\pm(0.01-0.02)$ mag for $\mathrm{B}-\mathrm{A}-\mathrm{F}-\mathrm{G}$ stars and of $\pm(0.02-0.03) \mathrm{mag}$ for $\mathrm{K}-\mathrm{M}$ stars.

The two methods used for the determination of spectral classes and absolute magnitudes give compatible results: the spectral classes agree typically to within 1 decimal subclass, the luminosity classes are usually the same in both cases.

The color excesses $E_{Y-V}$ were calculated as differences between the observed $Y-V$ and the intrinsic color indices $(Y-V)_{0}$ taken from Straižys (1992) for stars of various 


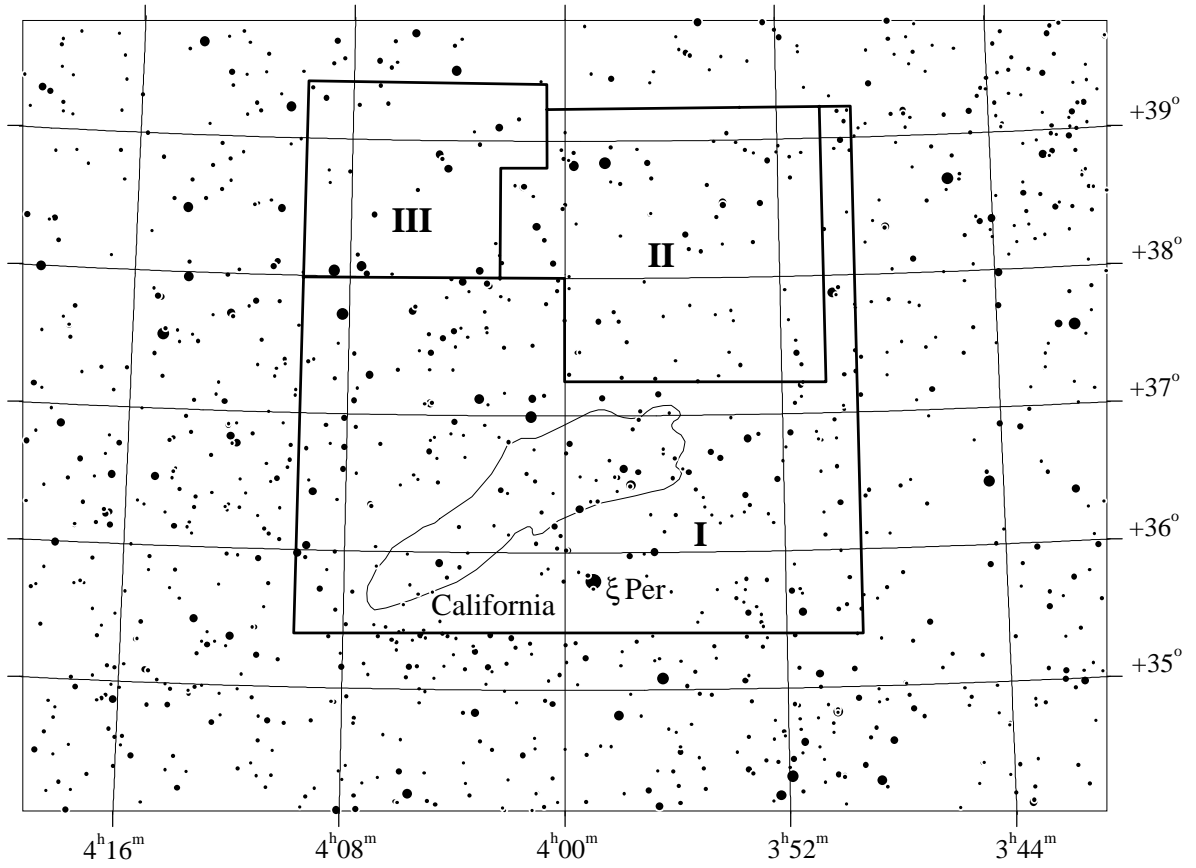

Fig. 1. The chart for the California Nebula area showing the boundaries of the investigated area and its division into three areas of different extinction. The coordinates are for 2000.0. spectral and luminosity classes. The distances of stars were derived as usual:

$5 \log r=V-M_{V}+5-R E_{Y-V}$,

where $R=4.16$ for the normal interstellar extinction law (Straižys et al. 1996).

The results of photometric quantification, i.e. spectral types, absolute magnitudes, color excesses $E_{Y-V}$, extinctions $A_{V}$, distances $r$ and the quantification accuracies $\sigma Q$, are given in Paper I. That paper also contains a comparison of distances determined from Vilnius photometry with those from the Hipparcos parallaxes up to 250 pc. The standard deviation of 23 stars in common is \pm 25 pc in distance and \pm 0.45 mag in absolute magnitude. No systematic difference is found between the photometric and trigonometric distances.

\section{Interstellar extinction}

According to the general trend of extinction with distance, the area can be divided into three smaller areas with boundaries shown in Fig. 1. The largest of the three, Area I, has a moderate surface density of background stars and embraces the California Nebula with its nearest surroundings. Area II is located north-west of the nebula and includes the dark clouds L 1449 and L 1456. Area III with the dark cloud L 1459 is located north-east of the nebula. In Areas II and III there are many spots exhibiting a low surface density of background stars, especially on the blue prints of the Palomar atlas.

Figure 2 shows the plot of the extinction versus distance for Area I. Here, up to $\sim 110$ pc distance, all stars are either unreddened or only slightly reddened. The first two really reddened stars are observed at a distance of $110 \mathrm{pc}$. More reddened stars start to appear at $\sim 140$ pc, but only

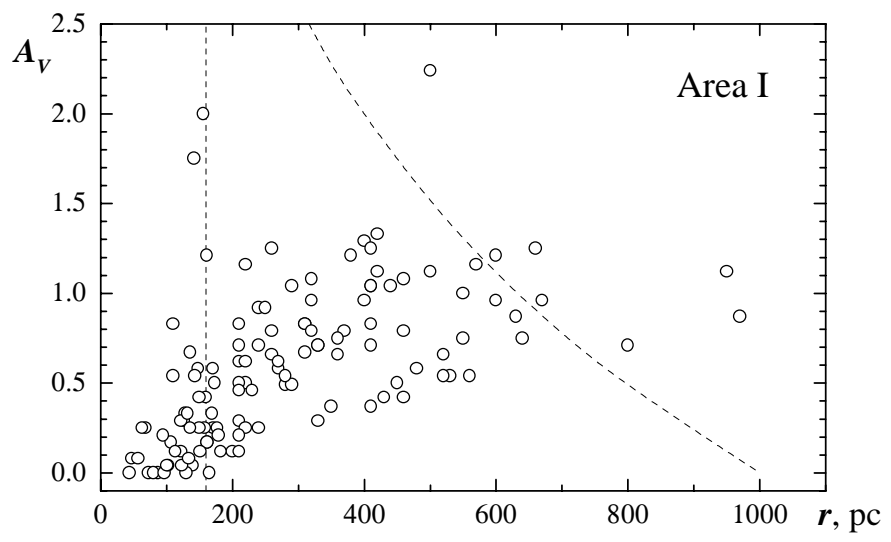

Fig. 2. Interstellar extinction $A_{V}$ plotted against distance $r$ in parsecs for Area I. The vertical broken line shows the accepted distance of the dust cloud at $160 \mathrm{pc}$. The broken curve corresponds to the limiting magnitude $V=11.0$ and $M_{V}=+1.0$; all stars to the right of the curve are either apparently fainter or absolutely brighter.

three of them exhibit extinctions larger than $1.7 \mathrm{mag}$. The extinction of the remaining stars in this area is lower than $1.3 \mathrm{mag}$. The stars in the distance range from $250 \mathrm{pc}$ to about $1 \mathrm{kpc}$ form, in Fig. 2, a belt between the $A_{V}$ values 0.3 and $1.3 \mathrm{mag}$, with a mean of $\sim 0.8 \mathrm{mag}$. This value can be considered as the mean extinction in the direction of the California Nebula.

There is no doubt that many more stars with $A_{V}>$ $1.3 \mathrm{mag}$ should be present in Area I, but due to considerable extinction they are apparently fainter than our approximate magnitude limit at $V=11 \mathrm{mag}$ (see the limiting curve for $V=11$ and $M_{V}=+1.0 \mathrm{mag}$, corresponding to A0 V and K III stars which are among the most luminous stars in the area). 


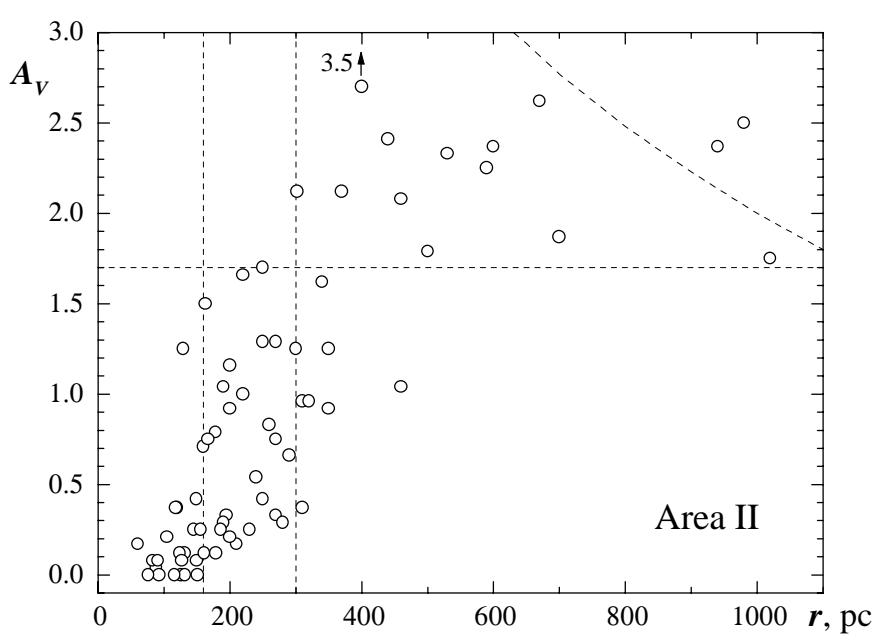

Fig. 3. Interstellar extinction $A_{V}$ plotted against distance $r$ in parsecs for Area II. The two vertical broken lines show the front edges of the dust clouds at distances of $160 \mathrm{pc}$ and $300 \mathrm{pc}$. The horizontal broken line corresponds to $A_{V}=1.7 \mathrm{mag}$, the maximum extinction of stars affected by the first dust layer. The broken curve near top right corresponds to the limiting magnitude $V=13$ and $M_{V}=+1.0$.

A sudden appearance of the reddened stars at $110 \mathrm{pc}$ means that somewhere beyond this distance the dust cloud begins. Due to the distance determination errors, the stars marking the front side of the cloud should be scattered within approximately $\pm 25 \%$, which at a distance of $160 \mathrm{pc}$ gives about $40 \mathrm{pc}$ (rms error). If the first two reddened stars appear at $110 \mathrm{pc}$ due to this scatter, the cloud may actually be situated as far as $150 \mathrm{pc}$.

On the other hand, the estimation of the distance to the cloud may be obtained from the observed distances of unreddened stars. In the direction of the area, the stars with very low extinction $\left(A_{V} \leq 0.2 \mathrm{mag}\right)$ are seen up to $210 \mathrm{pc}$. Subtracting the rms error of $40 \mathrm{pc}$, we obtain a distance of $170 \mathrm{pc}$ to the front edge of the cloud. The two estimates, 150 and 170 parsecs, are sufficiently close to each other, taking into account the fact that the closest reddened stars and the farthest unreddened stars can be occasionally found within the $3 \sigma$ error box (three standard deviations). Consequently, we are safe in considering the front edge of the cloud to be at $\sim 160 \mathrm{pc}$ distance. The absolute error of this distance probably cannot be larger than $\pm 20 \mathrm{pc}$, since in the opposite case it would be difficult to explain the presence of both the reddened stars at $110 \mathrm{pc}$ and the unreddened stars at $210 \mathrm{pc}$.

In Area I we do not find evidence of a possible second cloud at larger distances. Probably, the only cloud present is that at $160 \mathrm{pc}$. In this respect Area I seems to be very similar to the Per OB2 association area which contacts the California Nebula from the south (Černis 1993). A one-cloud structure is also found in some other areas in Taurus and Perseus (for further discussion, see Sect. 4). The dependence of interstellar extinction on distance for Area II is shown in Fig. 3. It is evident that up to $\sim 250 \mathrm{pc}$ the distribution of stars resembles that seen in Fig. 2. The first two considerably reddened stars appear at distances of 130 and $164 \mathrm{pc}$. This is in accordance with the model that all this area is covered by the extension of the dust cloud observed in the direction of the Per OB2 association and the California Nebula.

However, in Area II all stars (except one), which are farther than $350 \mathrm{pc}$, are affected by the extinction $A_{V} \geq$ $1.5 \mathrm{mag}$, and the stars with $r<300 \mathrm{pc}$ and $A_{V}>1.7 \mathrm{mag}$ are completely absent. Heavily reddened stars start to appear only at $300 \mathrm{pc}$. Another region in the graph of Area II with $r>350 \mathrm{pc}$ and $A_{V}<1.7 \mathrm{mag}$ is also empty, while in Fig. 2 the corresponding region is well populated. Both these features of the plot in Fig. 3 support the presence of a second dust cloud. It is not likely that such distribution of stars in the $A_{V}$ vs. distance plot would have been caused by selection effects.

Consequently, somewhere between $r_{1}=250 \mathrm{pc}$ (the minimum distance of stars with $A_{V}>1.5 \mathrm{mag}$ ) and $r_{2}=350 \mathrm{pc}$ (the maximum distance of stars with $A_{V}<$ $1.5 \mathrm{mag}$ ) the extinction jumps up by $\sim 1 \mathrm{mag}$. The most natural explanation of this feature seems to be the presence in Area II of the second dust cloud at $\sim 300 \mathrm{pc}$. At this distance its $25 \%$ error corresponds to about $75 \mathrm{pc}$, which is more than sufficient to explain the observed difference in limiting distances of stars with high and low extinction in Area II.

Almost all stars with $A_{V}>1.8 \mathrm{mag}$ are apparently fainter than $V=11 \mathrm{mag}$; with brighter limiting magnitude (as in Area I) they would be undetectable. Their spectral types are A2-A3 V-III and K2-K5 III.

Area III is covered by the dust cloud L 1459, and almost all of the stars observed in it are closer than the cloud and are therefore mostly unreddened. Probably, our limiting magnitude in this area was insufficient to reach the stars inside or behind the dust cloud. Only three stars with $A_{V}$ between 0.5 and 0.9 are found.

\section{Discussion}

Thus, we have found firm evidence that nearly all the investigated area is covered by a dust cloud at $\sim 160$ pc distance. As it was already noted in a previous section, this distance is close to that of the dust clouds in the Taurus and Perseus areas investigated by us earlier using the same method. The extinction versus distance diagram for Area I is found to be very similar to that obtained for the Per OB2 association area (Černis 1993), while the same diagram for Area II is similar to those for the areas of the reflection nebula NGC 1333 and the open cluster IC 348 (Černis 1990, 1993). The distances of the dust layers are compared in Table 2. Since the calibration of the Vilnius system in terms of absolute magnitudes in References 1-6 was based on the Hyades distance modulus of $3.2 \mathrm{mag}$, all the distances determined in these six papers were multiplied by 1.05 to adjust them to the new Hyades distance modulus of $3.3 \mathrm{mag}$. The table also includes the data for two areas observed by other authors, namely, the area with the dark cloud L 1478 in Perseus (Ungerer et al. 1985) and 


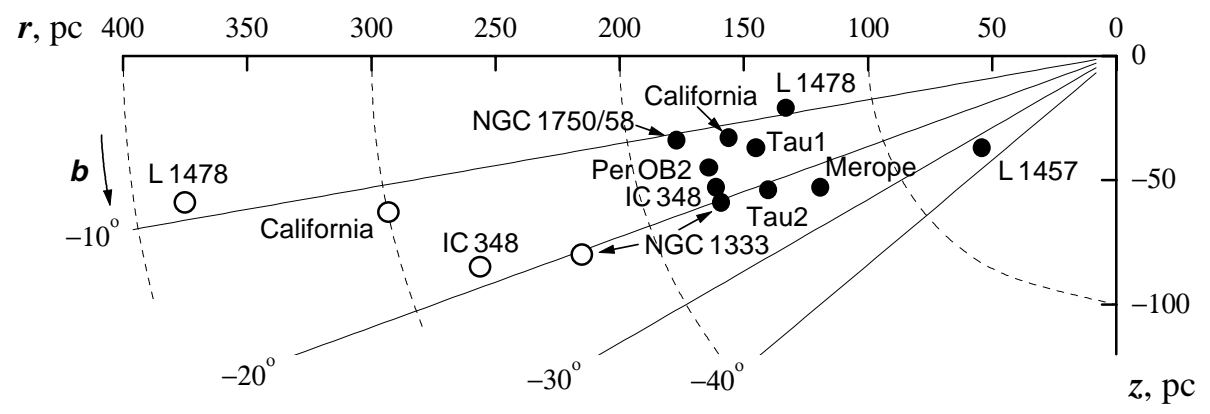

Fig. 4. The polar diagram "distance versus galactic latitude" for the Taurus, Perseus and Aries dust clouds. The dust clouds are shown by solid dots (the first layer) and open circles (the second layer).

Table 2. Distances of the dust layers in Taurus and Perseus determined by Vilnius photometry and transformed to the new distance modulus of the Hyades. Two additional areas, L 1478 and L 1457, are also included. Radial velocities determined from radio lines of $\mathrm{CO}$ are taken from Ungerechts and Thaddeus (1987).

\begin{tabular}{|c|c|c|c|c|c|c|}
\hline \multirow[b]{2}{*}{ Area } & \multicolumn{6}{|c|}{ Layers } \\
\hline & $\begin{array}{c}l \\
(\operatorname{deg})\end{array}$ & $\begin{array}{c}b \\
(\operatorname{deg})\end{array}$ & $\begin{array}{c}\mathrm{I} \\
(\mathrm{pc})\end{array}$ & $\begin{array}{l}\text { II } \\
(\mathrm{pc})\end{array}$ & $\begin{array}{c}R V \\
\left(\mathrm{~km} \mathrm{~s}^{-1}\right)\end{array}$ & Ref. \\
\hline Taurus 1 area & 174 & -14 & 150 & - & +6.3 & 1 \\
\hline Taurus 2 area & 178 & -21 & 150 & - & +7.0 & 2 \\
\hline Merope cloud & 167 & -24 & 130 & - & +9.7 & 3 \\
\hline NGC 1333 area in Per & 158 & -20 & 170 & 230 & +6.8 & 4 \\
\hline NGC $1750 / 58$ in Tau & 179 & -11 & 180 & - & +6.0 & 5 \\
\hline IC 348 area in Per & 160 & -18 & 170 & 270 & +8.5 & 6 \\
\hline Per OB2 area & 160 & -15 & 170 & - & +8.2 & 6 \\
\hline L 1478 cloud in Per & 163 & -9 & 135 & 380 & -2.7 & 7 \\
\hline L 1457 cloud in Ari & 159 & -34 & 65 & - & -1.2 & 8 \\
\hline California Nebula area & 160 & -12 & 160 & 300 & $\begin{array}{l}-5.0 \\
+3.1\end{array}$ & 9 \\
\hline
\end{tabular}

References: 1. Straižys \& Meištas (1980), 2. Meištas \& Straižys (1981), 3. Černis (1987), 4. Černis (1990), 5. Straižys et al. (1992), 6. Černis (1993), 7. Ungerer et al. (1985), 8. Hobbs et al. (1986), 9. This paper.

the area with the dark cloud L 1457 (MBM 12) in Aries (Hobbs et al. 1986). Both clouds probably belong to the same complex of dust and molecular clouds.

The two dust layer structure in Perseus has also been detected by other authors. Probably, the first indication of the two layers of the CO molecules in the dark cloud north of the California Nebula was found by Elmegreen \& Elmegreen (1978). Later on, Ungerer et al. (1985) investigated a dust concentration in the L 1478 molecular cloud located $3^{\circ}$ east of the nebula. From $U B V$ photometry and spectral classification data they identified two dust layers at distances of $135 \mathrm{pc}$ and $380 \mathrm{pc}$. The presence of two dust layers in Perseus was confirmed by Ungerechts \& Thaddeus (1987) from analysis of the available extinction investigations. They suspected that the Taurus dark clouds at $\sim 140 \mathrm{pc}$ distance extend into Perseus, while the molecular and dust clouds associated with the IC 348 cluster, NGC 1333 nebula and Per OB2 association are farther away. They accepted a distance of $350 \mathrm{pc}$ for the clouds associated with the California Nebula, as well as for the chain of molecular clouds extending from California towards Auriga. In some parts of these clouds, double or triple lines of the $\mathrm{CO}$ molecule were observed, pointing to the presence of two or more gas layers with different radial velocities. In the dust clouds north of the California Nebula Herbertz et al. (1991) found a complicated pattern of cloud fragments with average velocities near -7 , -3 and $+3 \mathrm{kms}^{-1}$. Wouterloot \& Habing (1985) have also found evidence for the two clouds from $\mathrm{OH}$ radio line observations.

Further evidence for the two layers at different distances in the Per OB2 association area was found by Krelowski et al. (1996) from analysis of the shapes of extinction curves in the ultraviolet, the strength ratio of two diffuse interstellar bands at 578 and $580 \mathrm{~nm}$, and the strengths of interstellar $\mathrm{CN}$ and $\mathrm{CH}$ molecular bands and sodium lines. They suspect that the two clouds have different absorbing properties. Sonnentrucker et al. (1999) have also found two clouds with different radial velocities observed in the $661 \mathrm{~nm}$ diffuse interstellar band. Kaczmarczyk (2000) recently identified two components of the interstellar $\mathrm{C}_{2}$ bands in the ultraviolet spectra of the star X Per, also corresponding to two gaseous layers with different radial velocities.

Černis (1993) has proposed a model of distribution of the two dust layers, based on his finding that the distance of the Perseus clouds increases with the apparent approach to the Galactic equator. This means that the complex of dust clouds in the second layer may be more or less parallel to the Galactic plane.

The same tendency is confirmed by the present paper, as can be seen from the polar diagram shown in Fig. 4. On this diagram the data for the seven areas investigated by our team earlier are plotted together with the new data for the California area. Additionally, the areas of the dark clouds L 1478 (Per) and L 1457 (Ari) are also shown. The clouds of the second layer form an elongated complex which is situated $60-80$ pc below the Galactic plane and is inclined by a small angle to the plane.

The clouds of the first layer concentrate in a formless group at $130-180$ pc from the Sun and 20-60 pc below the Galactic plane. The spread of these clouds in longitude is about 20 degrees. No correlation is found between the longitude and the distance from the Galactic plane. This dust layer may also be parallel to the plane. This is shown by the position in Fig. 4 of the Aries cloud L 1457 at $b=-34^{\circ}$. Its distance from the Sun, $\sim 65 \mathrm{pc}$, was 
estimated by Hobbs et al. $(1986,1988)$ and Hearty et al. (2000a). However, this distance is very uncertain and, according to Hearty et al. (2000b), may be somewhat larger (58-90 pc). Probably, the Aries cloud belongs to the same complex as the clouds of the first layer in Taurus, Auriga and Perseus, although its radial velocity is somewhat different (see Table 2).

It is known that the Sun is located about 10 pc above the Galactic plane. In this case, all the distances of the clouds from the Galactic plane should be reduced by this amount. According to Černis (1993) and de Zeeuw at al. (1999), the Per OB2 association starts at $338 \pm 16 \mathrm{pc}$ and $318 \pm 27 \mathrm{pc}$ distances, respectively. Thus, the distances of the association and the dust clouds north of California coincide within the distance determination errors. The distance of the second layer determined in the present paper, $300 \mathrm{pc}$, is smaller than $350 \mathrm{pc}$, the distance accepted by Ungerechts \& Thaddeus (1987). This means that their estimation of the mass of the Perseus clouds may be slightly overestimated.

Future investigations should help show where the clouds of both layers start and end. The second layer may either end abruptly at some distance from the Sun or may reach the Galactic plane. When approaching the Sun, both layers may merge at $\sim 150 \mathrm{pc}$ distance and at galactic latitude between $-20^{\circ}$ and $-30^{\circ}$. Therefore, additional investigations of the cloud complex at lower and higher latitudes are desired. On the other hand, we need more exact distances to the Aries dark clouds for the estimation of their relation to the Taurus-Auriga-Perseus complex. The investigation of the Aries region by our group is in progress. It is also important to verify if interstellar lines in the spectra of the heavily reddened early-type stars of Area II exhibit two components corresponding to the clouds with different radial velocities.

\section{References}

Bartašiūtè, S. 1999, Baltic Astron., 8, 443

Černis, K. 1987, Ap\&SS, 133, 355

Černis, K. 1990, Ap\&SS, 166, 315

Černis, K. 1993, Baltic Astron., 2, 214

Elmegreen, D. M., \& Elmegreen, B. G. 1978, ApJ, 219, 105
Hearty, T., Neuhäuser, R., Stelzer, B., et al. 2000a, A\&A, 353, 1044

Hearty, T., Fernandez, M., Alcala, J. M., Covino, E., \& Neuhäuser, R. 2000b, A\&A, 357, 681

Herbertz, R., Ungerechts, H., \& Winnerwisser, G. 1991, A\&A, 249,483

Hobbs, L. M., Blitz, L., \& Magnani L. 1986, ApJ, 306, L109

Hobbs, L. M., Blitz, L., Penprase, B. E., Magnani, L., \& Welty, D. E. 1988, ApJ, 327, 356

Kaczmarczyk, G. 2000, Acta Astron., 50, 151

Khavtassi, J. Sh. 1960, Atlas of Galactic Dark Nebulae (Abastumani Observatory, Tbilisi)

Krelowski, J., Megier, A., \& Strobel, A. 1996, A\&A, 308, 908

Lynds, B. T. 1962, ApJS, 7, 1

Meištas, E., \& Straižys, V. 1981, Acta Astron., 31, 85

Nikonov, V. B. 1976, Izvestija Crimean Obs., 54, 3

Perryman, M. A. C., Brown, A. G. A., Lebreton, Y., et al. 1998, A\&A, 331, 81

Sonnentrucker, P., Foing, B. H., Breifellner, M., \& Ehrenfreund, P. 1999, A\&A, 346, 936

Straižys, V. 1982, Pisma AZh, 8, 481

Straižys, V. 1992, Multicolor Stellar Photometry (Pachart Publishing House, Tucson)

Straižys, V., \& Meištas, E. 1980, Acta Astron., 30, 541

Straižys, V., \& Meištas, E. 1981, AZh, 58, 367

Straižys, V., Glagolevskij, Y. V., Romanyuk, I. I., \& Bychkov, V. D. 1982a, Pisma AZh, 8, 183

Straižys, V., Wisniewski, W. Z., \& Lebofsky, M. J. 1982b, Ap\&SS, 85, 271

Straižys, V., Kurilienè, G., \& Jodinskienè, E. 1982c, Bull. Vilnius Obs., No. 60, 3

Straižys, V., Černis, K., \& Hayes, D. S. 1985, Ap\&SS, 112, 251

Straižys, V., Černis, K., \& Meištas, E. 1992, Baltic Astron., 1, 125

Straižys, V., Černis, K., \& Bartašiūtè, S. 1996, Baltic Astron., 5,125

Straižys, V., Černis, K., \& Bartašiūtè, S. 2001, Baltic Astron., 10, 319, Paper I

Ungerechts, H., \& Thaddeus, P. 1987, ApJS, 63, 645

Ungerer, V., Mauron, N., Brillet, J., \& Rieu, N.-Q. 1985, A\&A, 146,123

Wouterloot, J. G. A., \& Habing, H. J. 1985, A\&AS, 60, 43

Zdanavičius, K. 1975, Bull. Vilnius Obs., No. 41, 3

Zdanavičius, K. 1996, Baltic Astron., 5, 549

Zdanavičius, K., \& Černienè, E. 1985, Bull. Vilnius Obs., No. 69,3

de Zeeuw, P. T., Hoogerwerf, R., \& de Bruijne, J. H. J. 1999, AJ, 117, 354 